\title{
.
}

Emilio López Viñuela*

\section{UN APUNTE SOBRE LA TRANSFERENCIA DE TECNOLOGÍA EN ISRAEL}

De la innovación y el progreso tecnológico depende en gran medida el bienestar material de las sociedades. Muchos países buscan por ello en la ciencia y en la tecnología un medio de impulsar su productividad y competitividad mediante la configuración de un sistema nacional, o ecosistema, de innovación.

Israel constituye en este ámbito un referente por el éxito alcanzado, sean los que sean los indicadores a los que se acuda. Una mirada a ese ecosistema, su evolución, la relación entre sus distintos elementos y su situación actual es, por ello, de gran interés.

En particular, el objetivo del artículo será examinar con un cierto detalle un único aspecto del ecosistema, cual es el de la transferencia de tecnología. Se analiza en qué consiste ese proceso de transferencia y qué papel tienen en Israel las oficinas de transferencia de tecnología. Se hace referencia, asimismo, a algunas organizaciones relevantes en el proceso, para finalizar con la mención de un conjunto de rasgos destacables del modelo israelí.

Palabras clave: innovación, progreso tecnológico, sistema nacional de innovación, ecosistema de innovación, transferencia de tecnología, oficinas de transferencia de tecnología.

Clasificación JEL: O32, O53.

\section{Introducción}

La innovación es un motor muy relevante de la productividad, el crecimiento y el desarrollo económico. Así lo muestra sin discrepancias la abundante literatura al respecto ya desde Schumpeter o de forma más reciente (ver, p. ej., Rosenberg, 2004 o Hausmann y Domínguez, 2020). El progreso tecnológico permite una producción más eficiente de más y mejores bienes y servicios, que es de lo que depende la prosperidad.

* Técnico Comercial y Economista del Estado. Versión de enero de 2022.

https://doi.org/10.32796/bice.2022.3143.7346
La innovación hace que aumente la competitividad de la economía al reducirse los costes de producción y mejorar la capacidad exportadora de las empresas. Permite reducir el déficit público y, en estos momentos en que las cadenas de valor están de algún modo distorsionadas, adquiere aún más importancia ser tecnológicamente independientes en algunos productos clave. Si se añade su papel fundamental en la (re)industrialización, hay que concluir que resulta muy necesario contar con un buen nivel tecnológico.

Para lograrlo se requiere una adecuada organización de un sistema nacional de $\triangle$ 
innovación o ecosistema de innovación. Un estructurado ecosistema de innovación ha de contar con una serie de elementos bien definidos y organizados, a la vez que cohesionados entre sí. En este artículo se comentan estos elementos de forma general, para centrarse en un aspecto en concreto, que es el de cómo se estructura la transferencia de tecnología, y estudiar específicamente el caso de Israel. Nos detendremos a analizar en qué consiste ese proceso de transferencia y cómo actúan en él las oficinas de transferencia de tecnología. Se hace referencia, asimismo, a algunas organizaciones que tienen un papel de importancia en el proceso, para finalizar con la mención a un conjunto de rasgos destacables del modelo israelí.

\section{Sistema nacional de innovación (ecosistema de innovación)}

El sistema nacional de innovación SNI o ecosistema de innovación, puede definirse (Buesa, 2012) como el conjunto de instituciones y empresas, y las relaciones entre ellas, que orientan su actividad total o parcialmente a hacer progresar la ciencia o aumentar la capacidad tecnológica de una economía.

No se va a entrar en consideraciones sobre cuestiones relativas a la organización de un SNI, pues, a nuestros efectos, es suficiente hacer una referencia general a los elementos que integran un ecosistema de innovación, que se resumen en lo siguiente.

\section{RECUADRO 1}

DEFINICIONES

Investigación: aquella actividad cuyo objetivo es la adquisición de nuevos conocimientos que puedan resultar de utilidad para la creación de nuevos productos, procesos o servicios o contribuir a mejorar considerablemente los ya existentes.

Desarrollo: la adquisición, combinación, configuración y empleo de conocimientos y técnicas ya existentes, de índole científica, tecnológica, empresarial o de otro tipo, con vistas a la elaboración de planes y estructuras o diseños de productos, procesos o servicios nuevos, modificados o mejorados. Se podrá incluir también la elaboración de proyectos, diseños, planos y demás tipos de documentación, siempre y cuando no vaya destinada a usos comerciales, así como el desarrollo de prototipos y proyectos piloto que puedan utilizarse comercialmente cuando sean necesariamente el producto comercial final.

Innovación: es la aplicación de un método de producción o suministro nuevo o significativamente mejorado, incluyendo cambios significativos en cuanto a técnicas, equipos y/o programas informáticos (innovación tecnológica y en materia de procesos), aplicación de nuevos métodos organizativos a las prácticas comerciales (innovación comercial), la organización del centro de trabajo o las relaciones exteriores de la empresa (innovación en materia de organización), etc.

Transferencia de conocimientos (de tecnología): proceso destinado a adquirir, recabar y compartir conocimientos expresos y tácitos, incluyendo, además de conocimientos científicos y tecnológicos, otros tipos de conocimientos, como conocimientos del uso de normas y reglamentos que los incorporan y de entornos de condiciones reales de funcionamiento y métodos de innovación en materia de organización, así como gestión de conocimientos relacionados con la identificación, adquisición, protección, defensa y explotación de otros activos inmateriales.

Fuente: Comisión Europea (2014) y OECD (2018). 
Conocimiento: sistema educativo y centros de investigación

Un SNI ha de contar, en primer lugar, con un entorno adecuado para la producción de ideas e investigación. Se requiere una población con un alto nivel de formación, en todos los ámbitos, pero son especialmente relevantes el científico e ingenieril. Es primordial, pues, contar con un buen sistema educativo, buenas universidades y centros de investigación, científicos y tecnológicos. Hay que mencionar, asimismo, la formación que se recibe en el seno de las empresas, ya que una parte de la investigación se lleva a cabo en ellas.

\section{Intervención del sector público}

En este ámbito es preciso el apoyo público. Tal apoyo público a la innovación se justifica ante la presencia de fallos de mercado que sin la intervención del Gobierno dan como resultado un equilibrio donde la inversión privada es subóptima desde un punto de vista social (Arrow, 1962). La inversión en investigación e innovación genera nuevos conocimientos cuya asimilación o adopción por parte de empresas que no asumieron los costos de desarrollo no puede evitarse por completo. El beneficio privado, que constituye la base para la toma de decisiones en las empresas, no incluye ese componente de desbordamiento, como tampoco diversas limitaciones sobre las decisiones de inversión de las empresas, de forma que la inversión privada tiende a ser más baja que la que se considera en un mercado óptimo.

El objetivo del apoyo gubernamental a la I+D es garantizar que la «producción» de conocimiento tenga lugar en un nivel óptimo desde el punto de vista social. Esto puede lograrse eliminando las limitaciones a la inversión o proporcionando apoyo directo para la ejecución de este tipo de actividades, lo que permite a las empresas obtener un rendimiento igual al rendimiento social y elegir sus inversiones en consecuencia.

La intervención del Gobierno tiene esencialmente tres componentes: proporcionar el entramado legal correspondiente (leyes de propiedad intelectual, de innovación, impositivas, etc.), dar apoyo financiero a la investigación básica y configurar acciones de colaboración universidad-industria.

Sendos análisis (Lach et al., 2008; MesserYaron, 2014) muestran la instrumentación del apoyo gubernamental a la innovación industrial en Israel y su favorable impacto sobre la economía israelí.

\section{Sistema de financiación: público y privado}

Un buen sistema de financiación es otro elemento decisivo. Hay que notar que en el mundo de la tecnología el riesgo es muy elevado y que muchos de los proyectos resultarán fallidos, por lo que el capital riesgo es determinante. La financiación en las fases iniciales tiene un carácter radicalmente distinto de la financiación bancaria de proyectos ya en marcha, por lo que se ha de contar con un bien instrumentado y adecuadamente dotado sistema de financiación, público y privado.

\section{El sector empresarial, incubadoras $y$ aceleradoras}

El sector empresarial es un elemento imprescindible, pero con diferencias según países en cuanto al mayor o menor grado de elaboración y difusión de tecnología en su estructura productiva. Mientras que la investigación básica y aplicada es compartida $\triangleright$ 
entre los centros de investigación y las universidades, la actividad de I+D realizada por el sector empresarial se centra en el desarrollo tecnológico dirigido al conocimiento concreto asociado a los problemas productivos. Esta última se lleva a cabo por entidades comerciales que se dedican a desarrollar un producto que se adapte a la demanda del mercado.

En el caso de una economía en la que sea reducido el número de empresas que recurran a su propia innovación para mejorar la competitividad, cobra importancia la innovación abierta, es decir, los mercados de tecnología donde las empresas pueden comprar tecnología, o encargar desarrollos.

Un ecosistema rodado se caracteriza por la abundante creación de startups, lo que se ve favorecido, además, por la existencia de una red de incubadoras y aceleradoras tecnológicas que no solo ofrecen financiación, sino otros servicios como un entorno de trabajo, asesoramiento tecnológico $\mathrm{y}$, de forma importante, contactos con potenciales socios, inversores y clientes.

\section{Sistema de trasferencia de tecnología}

La transferencia de tecnología se puede definir (TWI Innovation Network, 2021) como la transmisión o entrega de información de avances tecnológicos entre el propietario de la misma y un tercero interesado en ella.

Según la Organización Mundial de la Propiedad Intelectual, OMPI-WIPO (WIPO, 2012), las diversas definiciones de transferencia de tecnología «se refieren al proceso de transformar propiedad intelectual en productos». Abundando en lo anterior, menciona que la academia y la industria son dos culturas que difieren entre ellas pero que viven juntas. «El proceso que las une y posibilita la comercialización de bienes basados en la investigación académica se define como transferencia de tecnología».

\section{De la investigación a la innovación: la transferencia de tecnología}

Aunque el análisis en mayor detalle de los puntos anteriores tenga un indudable interés, y proporcione un detallado conocimiento de un determinado SNI, nuestro objetivo, y en lo que nos vamos a centrar, es profundizar en el proceso de transferencia de tecnología y, específicamente, en Israel.

La tecnología generalmente se transfiere a través de recursos legales, como la concesión de licencias de propiedad intelectual patentada a empresas externas o bien a empresas ad hoc de nueva creación, que pueden ser fundadas por el investigador, de modo que la accesibilidad de los desarrollos innovadores esté asegurada en un rango amplio en todo el mundo. Este fenómeno ayuda a explotar el conocimiento ya existente sobre los avances tecnológicos $y$, al mismo tiempo, proporciona un margen para nuevas innovaciones.

Ha de notarse cómo, por un lado, una cuestión central en la transferencia de tecnología es la protección de la propiedad intelectual asociada con las innovaciones desarrolladas en las instituciones de investigación; y, por otro, cómo tal transferencia ofrece una alternativa para rentabilizar la inversión realizada en la concepción y desarrollo de una invención patentable cuando no se puede, no se quiere o no compensa económicamente llevar a cabo su producción, total o parcial, y posterior distribución comercial, o su implantación en el proceso productivo.

Por su lado, la parte industrial licenciataria habrá de cumplir una serie de condiciones $\triangleright$ 
que pueden incluir la creación de un plan de desarrollo o marketing satisfactorio y proporcionar información sobre la capacidad de la empresa para desarrollar y comercializar la invención dentro de un periodo de tiempo especificado, así como los pagos a realizar a la entidad licenciante.

La importancia de la transferencia de tecnología (Lenagh, 2012) radica en que ayuda a convertir la propiedad intelectual todavía en épocas tempranas en herramientas para uso de la comunidad de investigación, o en bases para que nuevas plataformas, productos o servicios se conviertan en productos para uso público.

La transferencia exitosa de la tecnología da visibilidad a la institución de investigación, pues obtiene reconocimiento y aumenta su reputación por su potencial de investigación e innovación, y beneficia a sus socios industriales, que pueden reducir los costos en investigación y desarrollo al obtener licencias. Pero a la postre, el beneficiario final de la transferencia de tecnología es el público, que se beneficia tanto de los productos que llegan al mercado como de los empleos resultantes del desarroIlo, fabricación y venta de productos.

Como resultado de la complejidad potencial del proceso de transferencia de tecnología, muchas empresas, universidades y entes gubernamentales cuentan con oficinas de transferencia de tecnología (OTT) dedicadas a la identificación de investigaciones que tengan un potencial interés comercial y a llevar a cabo las estrategias adecuadas para su explotación. Existe un gran componente jurídico, pues se trata de regular cómo se reparten los beneficios de mercado de una innovación.

Los principales elementos que configuran el mecanismo de transferencia de tecnología son la academia y la industria. La academia contribuye a promover y generar conocimiento novedoso, mientras que la industria sintetiza dicho conocimiento en una entidad comercializable. Esta relación tiene un impacto significativo en las economías impulsadas por el conocimiento y las innovaciones, que posteriormente conducen a una sociedad avanzada.

Sin embargo, esta interacción no existe desde siempre (Origiin, 2021b). Las instituciones educativas y los sectores industriales tenían sus caminos separados y sus objetivos se oponían entre sí, siendo el primero rígido hacia las formas tradicionales de difusión del conocimiento y el segundo flexible en la adopción de formas de generar riqueza; las universidades estaban aisladas de los diversos aspectos de la sociedad, mientras que las organizaciones industriales estaban prácticamente involucradas en mejorar las condiciones económicas. No se concebía que su interacción pudiese ayudar al país en su crecimiento y desarrollo.

Una primera razón por la que estos dos sectores comenzaron a unirse fue la guerra. La mayoría de los avances tecnológicos que tuvieron lugar en el siglo $x x$ fueron en el ámbito militar. Esto hizo que cambiara la perspectiva y se generalizase la idea de que los científicos e investigadores utilizaran sus estudios para atender las necesidades de la sociedad. Otro factor fue la propagación de la educación como derecho básico de todos. Esto permitió que la educación no se limitara a unos pocos, sino que se presentara como un bien accesible para todos. La investigación y las innovaciones comenzaron a desarrollarse simultáneamente.

Los investigadores académicos que tenían la posibilidad de generar productos industriales buscaron protección patentando su invención, que luego fue licenciada a la industria, que generaría productos comercializables utilizando la invención y compartiría las ganancias con $\triangleright$ 
los propietarios de la patente mediante el pago de royalties. Esto condujo a la génesis del mecanismo de transferencia de tecnología cuyo objetivo final es negociar un contrato para establecer una relación comercial con la otra parte.

\section{La transferencia de tecnología en Israel. El papel de las oficinas de transferencia de tecnología (OTT)}

Israel, entendiendo desde hace tiempo la importancia de la tecnología y la innovación y cómo esos conceptos son un elemento esencial para su prosperidad, tomó en su momento la decisión estratégica fundamental de reactivar un sector basado en esas premisas. Israel dedica desde hace años algo más del $4 \%$ del PIB a invertir en innovación, lo que condujo a la creación de una prosperidad económica sostenible, dando lugar a una enorme productividad y valor económicos. El resultado es que Israel, fruto de su éxito en la innovación tecnológica, es considerada como una start-up nation, título de una obra del mismo nombre (Senor y Singer, 2009).

El ecosistema israelí tiene muy desarrollados y bien organizados todos los elementos que lo integran, fruto de la cuidadosa puesta en marcha de una serie de medidas a lo largo del tiempo. En 1969 se creó la Oficina del Científico Jefe (OCJ), un departamento del Ministerio de Economía que otorgaba subvenciones para fomentar la innovación. Las subvenciones podían llegar hasta el 50\% de los gastos aprobados incurridos para un determinado programa. A cambio, el beneficiario estaba obligado a pagar regalías a la OCJ a tasas que oscilaban entre el 3 y el $6 \%$ de los ingresos derivados de los productos que incorporasen los conocimientos técnicos desarrollados o derivados del programa hasta un total del $100 \%$ del monto de las subvenciones totales recibidas, más intereses.

La Oficina del Científico Jefe pasó a denominarse, desde el año 2016, Autoridad Nacional de Innovación (una entidad propia y no un departamento del Ministerio de Economía), y es responsable de llevar a cabo políticas gubernamentales que promuevan la investigación y el desarrollo industrial en Israel y de proporcionar financiación con base en planes de investigación y desarrollo.

Ha de mencionarse, asimismo, la Ley de Apoyo a la Investigación y Desarrollo del año 1984, modificada en varias ocasiones, pero sustancialmente en los años 2005 y 2015, que define los parámetros de la política estatal en este terreno y contiene un programa de incentivos financieros y medidas de apoyo que pueden recibir las empresas si cumplen con determinados criterios de elegibilidad.

Israel cuenta además con una serie de leyes para fomentar la innovación tecnológica que incluyen, entre otras, la promoción de la competencia, la regulación de visados, impuestos y patentes, el fomento de la inversión en determinadas industrias innovadoras y la atracción y mantenimiento del capital riesgo en el país. Una visión sintética de la configuración del ecosistema de innovación israelí, desde sus inicios hasta el momento actual, puede verse en Research Office Legislative Council Secretariat, 2017a y Origiin, 2021b.

Pero, específicamente, el sistema de trasferencia de tecnología es considerado por la Organización Mundial de Propiedad Intelectual (WIPO, 2012) un modelo de mejores prácticas. Incluso economías con ecosistemas de innovación desarrollados miran a Israel en busca de un modelo en el que inspirarse (Dillon, 2019), pues este país ha alcanzado $D$ 
resultados verdaderamente notables en esta área en relación con su tamaño.

Las oficinas de transferencia de tecnología de Israel han generado más ingresos por las ventas de propiedad intelectual que cualquier otro país fuera de los EE. UU. Según el Central Bureau of Statistics de Israel, en el año 2019, tales ingresos ascendieron a 1.300 millones de NIS (unos 325 millones de euros). El $89 \%$ de los ingresos provinieron de las empresas asociadas a universidades. Otro dato revelador (Klein Leichman, 2018) es que el presupuesto de investigación del Technion, de aproximadamente 90 millones de dólares en ese año, era una fracción del MIT y, sin embargo, sus ingresos por la comercialización de la investigación eran muy similares.

Algunos autores (Yablonko, 2019) consideran que más relevante que generar ingresos por la transferencia de tecnología es la propia transferencia o diseminación del conocimiento a la industria y la sociedad. Indicadores en ese sentido serían, por ejemplo, las 628 solicitudes de patentes que las oficinas de transferencia de tecnología de Israel presentaron en 2020 y su participación en dicho año en el surgimiento de 75 empresas de nueva creación.

En los últimos años, y en consonancia con los resultados mencionados, tanto Harvard como la Universidad de California han elegido a israelíes experimentados para dirigir sus oficinas de transferencia de tecnología.

Los principales organismos encargados de la relación universidad-empresa en Israel son las oficinas de transferencia de tecnología (OTT), empresas subsidiarias de la universidad/organismo investigador, que tienen como principal objetivo lanzar al mercado proyectos de investigación en fase muy avanzada. En las OTT hay investigadores, pero, dado su carácter multidisciplinar, cuentan asimismo con economistas, ingenieros, comerciales, científicos, abogados expertos en patentes, en contratos, en propiedad intelectual, etc. Tener profesionales bien formados en estos campos, y de forma más concreta en la comercialización, es algo obligado, como lo es también disponer de las adecuadas herramientas y plataformas que faciliten su función de avanzar productos hacia el mercado.

Las universidades tienen regulaciones internas tanto sobre los derechos y obligaciones de los investigadores en lo concerniente a la transferencia de tecnología como sobre sus acuerdos con las OTT.

\subsection{La «brecha de desarrollo» y el proceso de transferencia de tecnología}

La dificultad mayor para transformar una invención en un producto útil es la llamada «brecha de desarrollo", que se encuentra entre el estado de inmadurez del descubrimiento académico y los requisitos probados de factibilidad de la industria. En Israel se ha prestado especial atención a salvar esta brecha.

El proceso de transferencia de tecnología gestionado por las OTT universitarias generalmente comprende las siguientes etapas (Research Office Legislative Council Secretariat, 2017b):

1. Un investigador académico informa a la OTT de su universidad sobre cualquier invención que piensa puede tener potencial comercial mediante un formulario de divulgación de innovación; es decir, se parte de una investigación académica básica y el entero proceso de transferencia de tecnología comienza con el investigador y su iniciativa. 
2. La OTT en cuestión evalúa el potencial de la invención tanto para su eventual registro como patente como para su comercialización.

3. Si el examen resulta positivo y se considera que la invención tiene valor comercial, la OTT solicita el registro de la patente y elabora una estrategia de marketing para la invención; luego tratará de identificar al socio comercial potencial (o licenciatario) interesado en la invención, negociará el acuerdo de licencia con ese socio y dará seguimiento al acuerdo. Los ingresos generados por los pagos de derechos de licencia y/o royalties por parte del licenciatario se comparten entre los inventores y la universidad de investigación. En la mayoría de las universidades de Israel, los inventores reciben el $40 \%$ de los ingresos por comercialización. Por otro lado, un porcentaje revierte para financiar investigación, normalmente el $20 \%$, y el $40 \%$ restante se destina a cubrir los gastos de la OTT y de la universidad.

En muchos casos, sin embargo, la invención es prematura para su comercialización y, por ello, se necesita un puente sobre la brecha de desarrollo. En ese caso, los expertos de la OTT identifican la «información faltante» e indican al investigador posibles vías de financiación para llevar a cabo las actividades requeridas para conseguir esa información.

El concepto de transferencia de tecnología nació en la Universidad de Wisconsin en 1925, y luego se nutrió y refinó en Israel, donde sus tres principales universidades crearon compañías con el objetivo de impulsar y gestionar su actividad de transferencia de conocimiento allá por los años sesenta: el Weizmann Institute creó Yeda en 1959, la Hebrew University creó Yissum en 1964 y la Tel Aviv University creó Ramot en 1973.

La primera OTT de su tipo fue iniciada por el Instituto de Ciencia Weizmann con el nombre de Yeda Research \& Development Co. Ltd. Su éxito ha contribuido a un cambio de paradigma en el modelo de transferencia de tecnología en Israel y, al mismo tiempo, se ha convertido en una vanguardia para otras OTT que se establecieron posteriormente. También ha establecido un punto de referencia al estar entre las mejores universidades del mundo en términos de generar ingresos a partir de la investigación académica comercializada. Las facultades de la universidad también actúan como consultoras de los sectores privados que ayudan a crear un vínculo entre el sector académico y el industrial. Su estrategia de crear un equilibrio armonioso entre el interés público y las ganancias financieras ha hecho que su modelo de transferencia de tecnología sea ejemplar para el resto del mundo.

En definitiva, el papel de la OTT incluye, pero no se limita (Origiin, 2021a), a lo siguiente:

- Verificar si la invención califica para el registro de patente.

- Deducir la probabilidad de comercializar una determinada innovación.

- Formular estrategias de mercado para introducir una innovación adecuada.

- Proponer la innovación a organismos industriales específicos que estén dispuestos a introducir el producto resultante en el mercado.

- Facilitar, a la universidad y a la entidad industrial interesada, el contrato de licenciamiento, en caso de que dicha propuesta se implemente con éxito. 
- Identificar cualquier falta en la investigación respecto a la innovación registrada que pueda obstaculizar la viabilidad comercial de la invención en cuestión.

- Brindar una orientación adecuada al investigador sugiriendo formas que puedan llenar el vacío creado por la brecha de investigación, de modo que la innovación se vuelva compatible con los estándares comercializables.

\subsection{Relación de OTT en Israel}

En este epígrafe se mencionan las OTT actualmente operativas en Israel, que tienen unas actividades similares en línea con lo ya mencionado antes para estas entidades. En los casos más destacados, se hace una breve descripción de sus rasgos y actividades, pero en otros únicamente se citan.

Las OTT y sus correspondientes entidades de investigación se analizan a continuación.

\section{Instituto Weizmann: Yeda Research and Development Company Ltd}

El Weizmann Institute of Science, ubicado cerca de Tel Aviv, es uno de los centros de investigación multidisciplinar más importantes del mundo. Yeda Research and Development, su empresa comercializadora, creada en 1959, ha patentado más de 160 tecnologías en los últimos dos años y lleva creadas más de veinte empresas spin-off basadas en su conocimiento.

Entre sus principales actividades se encuentran la identificación de proyectos de investigación con un potencial comercializador; la protección de la propiedad intelectual (PI) del instituto y de sus científicos; otorgar licencias de tecnología a la industria, así como la gestión de programas de financiación.

Dos de sus patentes, Copaxone ${ }^{\circledR}$ y

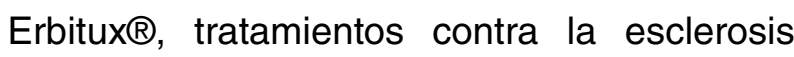
múltiple y el cáncer, respectivamente, han logrado sobrepasar los 1.000 millones de dólares de facturación.

Según los datos más recientes de Weizmann, Yeda ha registrado cerca de 2.000 familias de patentes y han surgido 73 spin-offs, generando un acumulado de 28.000 millones de dólares en ventas. El primer acuerdo exitoso de Yeda fue la licencia del medicamento para la esclerosis múltiple, Copaxone, a Teva Pharmaceuticals, en 1987.

El Instituto Weizmann tiene una política claramente establecida sobre transferencia de tecnología (WIPO, 2012) que incluye cuatro principios. El primero establece que la financiación privada de la investigación académica debe tener un alcance y un tiempo limitados a fin de garantizar al investigador otras actividades que le permitan una completa libertad de investigación.

El segundo principio se refiere al derecho de publicación. En cualquier forma en que se organice la transferencia de tecnología, el Instituto Weizmann se asegura de que el investigador mantenga su derecho a publicar artículos y trabajos sin limitaciones. El financiador no puede limitar el derecho a publicar.

El tercer principio fija que, al firmar acuerdos con una empresa industrial, la empresa se comprometerá a desarrollar diligentemente el producto para introducirlo en el mercado. Es decir, el Instituto Weizmann no permite que una empresa adquiera los derechos de tecnología con el fin de obtener una ventaja sobre los competidores simplemente impidiéndoles el acceso. Si la empresa industrial no desarrolla el producto, se rescinde su licencia. 
El cuarto principio establece que el Instituto Weizmann se reserva el derecho de comercializar otras tecnologías, incluso si pueden competir con una tecnología que ya ha sido comercializada a una determinada empresa.

La propiedad de la patente puede variar dependiendo del sistema que se haya utilizado para financiar la investigación y los diferentes acuerdos que se hayan alcanzado. Pero es regla fundamental del Instituto de Ciencias Weizmann mantener el título sobre la propiedad intelectual en el instituto y licenciarla en condiciones que garanticen el interés público, lo que, junto a los cuatro principios básicos mencionados, constituye en esencia principios éticos establecidos en función del interés general y no solo de su beneficio económico inmediato. El éxito del Instituto Weizmann como líder, tanto científica como comercialmente, demuestra que la protección del interés público no entra en conflicto con la transferencia de tecnología con éxito y que tal vez incluso vayan de la mano.

\section{Universidad Hebrea de Jerusalén: Yissum Research and Development Company}

Yissum se encarga de transferir la tecnología de la investigación desarrollada en la Universidad Hebrea de Jerusalén. Desde 1964, Yissum ha comercializado productos que generan un volumen de ventas de aproximadamente 2.000 millones de dólares al año, principalmente en sectores relacionados con la nanotecnología, medicina y farmacia, agricultura y nutrición, tecnologías del agua, ciencias informáticas y seguridad nacional.

Yissum es la OTT más grande de Israel en términos de patentes (más de 10.000), licencias (900) y empresas derivadas (125, incluidas destacadas como Mobileye y BriefCam) en una amplia variedad de campos. En la industria mundial de semillas, el tomate cherry de larga duración desarrollado en la Universidad Hebrea es un ejemplo principal.

\section{Universidad de Tel Aviv (TAU): Ramot}

Ramot es la empresa encargada de comercializar y proteger las invenciones que desarrollan los estudiantes, profesores e investigadores en la Universidad de Tel Aviv (TAU). Esta empresa provee un marco comercial y legal para las invenciones, protegiendo los descubrimientos con patentes y trabajando con la industria para acercar las innovaciones científicas al mercado.

Al igual que otras OTT, entre sus principales objetivos se encuentran: proteger las invenciones mediante el otorgamiento de patentes, maximizar el potencial comercial de las tecnologías desarrolladas en la TAU, incrementar la ratio de transferencia de tecnología desde los laboratorios hacia la industria, generar nuevas fuentes de ingresos para la universidad y sus inventores a través de la otorgación de licencias, dar apoyo a la generación de startups basadas en las tecnologías de la TAU y fomentar el emprendimiento tecnológico en la universidad.

A esas OTT fueron añadiéndose otras como:

- Instituto Tecnológico de Israel (Technion): T3 Technion Technology Transfer.

- Ariel University: ASI - Ariel Scientific Innovations Ltd.

- Universidad Bar-llan: BIRAD.

- Universidad de Ben-Gurion: BG.Negev Technologies \& Applications Ltd.

- Universidad de Haifa: Carmel-Haifa University Economic Corp. 
Algunas organizaciones de salud israelíes también tienen OTT; así:

- Hadassah Medical Organización: Hadasit. Fue fundada en 1986 como la primera OTT hospitalaria de Israel.

- MIGAL Galilee Research Institute: Gavish Galilee Bioapplications.

- Clalit Health Services: Mor Research Applications.

- Tel Aviv Medical Center: Tel Aviv Sourasky Medical Center.

- Shaare Zedek Medical Center: Shaare Zedek Scientific Ltd.

- Rappaport Research Institute: BioRap Technologies Ltd.

- Sheba Medical Center: Tel Hashomer Medical Research, Infrastructure and Services Ltd.

\section{Organizaciones relevantes en este ámbito}

Una contribución importante a la consecución de un modelo de transferencia de tecnología tan avanzado en Israel se atribuye a dos organizaciones.

Israel Tech Transfer Network (ITTN), Red de Transferencia de Tecnología de Israel. Es una organización privada sin fines de lucro que proporciona una plataforma para empresas de transferencia de tecnología y actúa como coordinadora de tales empresas. Sus objetivos son:

- Representar el interés de sus organizaciones miembros ante la Knesset (Parlamento de Israel), autoridades gubernamentales, ministerios, agencias y comités.
- Apoyar los esfuerzos de colaboración entre la comunidad de transferencia de tecnología en Israel y sus contrapartes en todo el mundo.

- Promover el conocimiento de las innovaciones y propuestas de investigación llevados a cabo en las principales universidades e institutos de investigación de Israel, mejorando así la accesibilidad del público a las mismas.

Actualmente, son quince las organizaciones asociadas (mencionadas en el punto anterior) e ITTN tiene la intención de agregar más miembros de los centros médicos e instituciones de investigación propiedad del Gobierno de Israel.

Israel Advanced Technology Industries (IATI), otro actor importante en este campo. IATI es la organización paraguas de Israel de las industrias de alta tecnología, ciencias de la vida y otras tecnologías avanzadas, cuyo objetivo es promover estas industrias y posicionarlas como líderes comerciales israelíes y mundiales.

Apoya y aboga por políticas públicas que fomenten la innovación y el espíritu empresarial israelíes y representa a Israel en acuerdos de cooperación estratégica con varios países y regiones extranjeras para promover la innovación y los intereses comerciales mutuos, y generan activamente oportunidades de negocio globales para sus miembros.

Sus foros y comités se reúnen regularmente para intercambiar ideas y lanzar proyectos para apoyar los intereses de la industria, tales como políticas fiscales, protección de la propiedad intelectual, educación STEAM ${ }^{1}$, etc.

El sistema STEAM consiste en educar en cinco materias (ciencia, tecnología, ingeniería, artes y matemáticas) con un sistema de aprendizaje conjunto y como un lenguaje cotidiano que se aplique a situaciones de la vida real de manera unificada. 
En la actualidad, tiene una plétora de miembros que incluyen instituciones académicas, incubadoras, entidades de inversión, empresas multinacionales proveedoras de servicios, oficinas de transferencia de tecnología, etc., de forma que, a través de esta amplia gama de miembros, la IATI conecta el ecosistema tecnológico de Israel, proporciona soluciones y apoyo en todos los niveles, e integra los diversos sectores de la industria con los objetivos gubernamentales estratégicos.

Ha de mencionarse, asimismo, en el contexto más amplio del ecosistema de innovación, la Start-Up Nation Central, que es una organización independiente cuyo objetivo es conectar la innovación israelí con los mercados exteriores. Además de con otros medios y actividades, cuenta para ello con una amplia base de datos, la Start-Up Nation Finder.

\section{Algunas consideraciones finales}

Una primera cuestión a destacar es la importancia de contar con un buen ecosistema de emprendimiento en el que no solo se produzca conocimiento, sino que se cuente con vías para convertir ese conocimiento en productos con valor de mercado.

Israel tomó partido decididamente por ello, de forma que se fueron definiendo los distintos elementos de un ecosistema entre los que hay que contar las oficinas de transferencia de tecnología, que han desempeñado y desempeñan un papel fundamental.

Estas oficinas son instituciones de carácter multidisciplinar, ni entidades de investigación ni entidades de comercialización, de forma que esto condiciona y ha de quedar reflejado en la formación de quienes trabajen en ellas, que proceden de diversos campos.
Un elemento destacable en el modelo de transferencia de tecnología israelí es que los investigadores no han de renunciar a su vocación científica sin que por ello queden excluidos de poder participar en los beneficios que se obtengan de la comercialización de sus invenciones.

Finalmente, y aunque queda fuera del objeto de este trabajo, sin hacer ninguna comparativa con el modelo español de transferencia de tecnología, se mencionan en la bibliografía algunas publicaciones e informes que permiten conocer el modelo y que incluyen en algunos casos propuestas de eventuales modificaciones y medidas a introducir para su mejora (Buesa, 2012; Conesa, s.f.; Larraga, 2019; Grau, 2019; Fundación CYD y Redtransfer, 2019; Rodríguez-Pomeda y Casani, 2007).

\section{Bibliografía}

Arrow, K. J. (1962). Economic welfare and the Allocation of Resources for Invention. In Universities-National Bureau Committee for Economic Research, Committee on Economic Growth of the Social Science Research Council (Ed.), The Rate and Direction of Inventive Activity: Economic and Social Factors, (pp. 609-626). Princeton University Press. https://www.nber.org/system/files/chapters/c2144/c2144.pdf

Buesa, M. (2012). El Sistema Nacional de Innovación en España: un panorama. Información Comercial Española (ICE), (869). http://www.revistasice.com/index.php/ICE/article/view/1548/1548

Comisión Europea. (2014). Comunicación de la Comisión, de 27 de junio de 2014. Marco sobre ayudas estatales de investigación y desarrollo e innovación (2014/C 198/01). Diario Oficial de la Unión Europea. https://eur-lex.europa.eu/legalcontent/ES/TXT/PDF/?uri=CELEX:52014XC 0627(01)\&from $=E L$ 
Conesa, F. (s.f.). Tópicos y realidades sobre la transferencia de conocimiento en España. Redtransfer. https://www.redtransfer.org/blog/topicosy-realidades-sobre-transferencia-de-conocimiento-en-espana/

Conesa, F. (s.f.). Transferencia versus transmisión del conocimiento: los nuevos sexenios. Redtransfer. https://www.redtransfer.org/blog/transferencia-versus-transmision-de-conocimientolos-nuevos-sexenios/

Conesa, F. (s.f.). ¿Tercera misión o tercera división? Redtransfer. https://www.redtransfer.org/blog/ tercera-mision-o-tercera-division/

Conesa, F. (s.f.). 25 años de Oficinas de Transferencia en España. Redtransfer. https://www.redtransfer.org/blog/25-anos-de-oficinas-detransferencia-en-espana/

Dillon, F. (2019, May 30). Ireland can learn from Israel's technology transfer model. The Irish Times. https://www.irishtimes.com/business/innovation/ ireland-can-learn-from-israel-s-technologytransfer-model-1.3907208

Fundación CYD y Redtransfer. (2019). Mejorar la creación de spin-off y las licencias de patentes en las universidades españolas. Informe CYD 2019. https://www.redtransfer.org/sites/default/files/Monograf\%C3\%ADa\%20ICYD\%202019\%20 $\% 281 \% 29$.pdf

Grau, C. (22 de octubre de 2019). La transferencia tecnológica, una puerta para la innovación. Do Better. By Esade. https://dobetter.esade.edu/es/ transferencia-tecnologia-innovacion?_wrapper_ format=html

Hausmann, R., \& Domínguez, J. (2020). Knowledge, Technology and Complexity in Economic Growth. Real Colegio Complutense. Harvard University. https://rcc.harvard.edu/knowledgetechnology-and-complexity-economic-growth

Israel Advanced Technology Industries (IATI). https://www.iati.co.il/

Israel Tech Transfer Network (ITTN). http://www.ittn.org.il
Klein Leichman, A. (2018, January 18). Why Israel rocks at commercializing academic innovations. Israe/21c. https://www.israel21c.org/whyisrael-rocks-at-commercializing-academic-innovations/

Lach, S., Parizat, S., \& Wasserteil, D. (2008). The impact of government support to industrial R\&D on the Israeli economy. E.G.P. Applied Economics Ltd. Research and Consulting in Economics, Marketing and Social Sciences. https://financedocbox.com/Investing/121596421-The-impact-of-governmentsupport-to-industrial-r-d-on-the-israeli-economy-final-report-english-translation-from-hebrew.html

Larraga, V. (Coord.) (2019). 2. ${ }^{\circ}$ Informe Ciencia y Tecnología en España 2019. Fundación Alternativas. https://www.fundacionalternativas.org/ las-publicaciones/informes/2o-informe-sobrela-ciencia-y-la-tecnologia-en-espana

Lenagh, A. (18 de diciembre de 2012). La importancia de la transferencia de tecnología. UNeMed. https://www.unemed.com/blog/the-importanceof-technology-transfer

Messer-Yaron, H. (2014). Technology Transfer in Israel - From bottom up to top down? 6th Meeting of the European TTO Circle. Council for Higher Education. https://ec.europa.eu/assets/jrc/events/ 20140120-tto-circle/jrc-20140120-tto-circle-messer.pdf

OECD. (2018). Oslo Manual 2018. Guidelines for collecting, reporting and using data on innovation. https://www.oecd.org/sti/inno/oslo-manual-2018-info.pdf

Origiin. (2021a). Technology transfer model: Israel. https://www.origiin.com/2021/08/06/technologytransfer-model-israel/

Origiin. (2021b). Israel Model of Fostering Innovation. https://www.origiin.com/2021/06/18/israelmodel-of-fostering-innovation/

Research Office Legislative Council Secretariat. (2017a). Fact Sheet. Innovation and $D$ 
technology industry in Israel. FSC23/16-17. https://www.legco.gov.hk/research-publications/ english/1617fsc23-innovation-and-technology-industry-in-israel-20170712-e.pdf

Research Office Legislative Council Secretariat. (2017b). Fact Sheet. Innovation and technology development in Israel. FS05/16-17. https://www. legco.gov.hk/research-publications/english/ 1617fs05-innovation-and-technology-development-in-israel-20170320-e.pdf

Rodríguez-Pomeda, J. y Casani Fernández de Navarrete, F. (2007). La transferencia de tecnología en España: diagnóstico y perspectivas. Economía Industrial, (366), 15-22. https://www.researchgate.net/publication/28201141_La_ transferencia_de_tecnologia_en_Espana_diagnostico_y_perspectivas

Rosenberg, N. (2004). Innovation and Economic Growth. OECD. https://www.oecd.org/cfe/tourism/34267902.pdf
Senor, D., \& Singer, S. (2009). Start-up Nation. The Story of Israel's Economic Miracle. Random House Digital, Inc. https://hadinur1969.files.wordpress.com/2017/11/dan_senor_saul_singer-startup_nation_the_story_of.pdf

Start-up Nation Central. https://www.startupnationcentral.org/

TWI Innovation Network. (2021). What is technology transfer (definition and examples). https://www. twi-global.com/technical-knowledge/faqs/ what-is-technology-transfer

WIPO. (2012). Technology Transfer in Countries in Transition: Policy and Recommendations. https:// www.wipo.int/edocs/pubdocs/en/wipo_pub_ transition_2_b.pdf

Yablonko, Y. (2019, Sep 5). Tech transfer is about more than revenue. Globes. https://en.globes. co.il/en/article-tech-transfer-is-about-morethan-revenue-1001299678 